\title{
Obesity Moderates the Effects of Motivational Interviewing Treatment Outcomes in Fibromyalgia
}

\author{
Anthony S. Kaleth, $\mathrm{PhD}^{1}$; James E. Slaven, $\mathrm{MS}^{2}$; and Dennis C. Ang, MD, $\mathrm{MS}^{3}$ \\ Department of Kinesiology, Indiana University-Purdue University Indianapolis, IN ${ }^{1}$; Department \\ of Biostatistics, Indiana University, Indianapolis, $\mathrm{IN}^{2}$; Division of Rheumatology, Department of \\ Medicine, Wake Forest University School of Medicine, Winston-Salem, NC ${ }^{3}$
}

\section{Corresponding Author: \\ Dennis Ang, MD, MS \\ Section of Rheumatology and Immunology \\ Wake Forest University Baptist Medical Center \\ Medical Center Boulevard, Winston-Salem, NC 27157 \\ Phone: 336-713-4264; Fax: 336-716-9821; Email: dang@wakehealth.edu}

\section{Funding Support:}

National Institute of Arthritis \& Musculoskeletal and Skin Diseases (1RO1AR054324-01A1)

ClinicalTrials.gov Identifier: NCT00573612

\section{Running Head}

Obesity and MI in Fibromyalgia

\section{Key Words}

Fibromyalgia; Motivational Interviewing; Physical Activity; Exercise; Obesity

Word Count: 2,752

\section{Conflict of Interest}

No professional relationships or conflicts of interest exist with any companies or manufacturers who will benefit from the results of the present study.

This is the author's manuscript of the article published in final edited form as: 


\begin{abstract}
Objective: Obesity is a common comorbid condition among patients with fibromyalgia (FM).

Our objective was to assess if obesity moderates the treatment benefits of exercise-based motivational interviewing (MI) for FM.
\end{abstract}

Methods: This is a secondary data analysis of a completed clinical trial of 198 FM patients who were randomized to receive either MI or attention control (AC). Using body mass index (BMI) to divide participants into obese $\left(\mathrm{BMI}>30 \mathrm{~kg} / \mathrm{m}^{2}\right)$ and non-obese $\left(\mathrm{BMI}<30 \mathrm{~kg} \mathrm{\textrm {m } ^ { 2 }}\right)$ groups, mixed linear models were used to determine interaction between treatment arms and obesity status with regards to the primary outcome of global FM symptom severity (Fibromyalgia Impact Questionnaire, FIQ). Secondary measures included pain intensity (Brief Pain Inventory, BPI), 6-minute walk test, and self-reported physical activity (Community Health Activities Model Program for Seniors).

Results: Of the 198 participants, 91 (46\%) were non-obese and 107 (54\%) were obese. On global FM symptom severity (FIQ), the interaction between treatment arms and obesity status was significant $(\mathrm{p}=0.02)$. In the non-obese group, MI was associated with a greater improvement in FIQ than AC. In the obese group, MI participants reported less improvement in FIQ compared to AC. The interaction analysis was also significant for BPI pain intensity $(\mathrm{p}=0.01)$, but not for the walk test and self-reported physical activity.

Discussion: This is the first study to show that obesity negatively affects the treatment efficacy of MI in patients with FM. Our findings suggest that exercise-based MI may be more effective if initiated after weight loss is achieved.

Copyright (C) 2017 Wolters Kluwer Health, Inc. Unauthorized reproduction of the article is prohibited. 


\section{INTRODUCTION}

Fibromyalgia (FM) is a highly prevalent chronic pain disorder characterized by a wide range of disabling symptoms, including fatigue, anxiety, cognitive impairment, and reduced physical functioning $[1,2]$. The complex clinical presentation of the disorder often complicates the diagnosis and treatment approach for clinicians, since many patients also present with a myriad of comorbid chronic conditions (e.g. sleep disorders, depression, hypertension, and type 2 diabetes) $[3,4]$. As the disorder worsens, many patients experience a progressive decline in physical activity [5], which likely contributes to excessive weight gain and worsening pain symptoms $[6,7]$.

Obesity is one of the most consistent comorbid conditions among patients with FM [8-

10]. The pathways linking chronic pain and obesity are multifactorial, involving both physiological and psychological mechanisms [11]. Nevertheless, the available research is persuasive that obesity has a powerful and independent influence on pain in patients with FM. Compared to normal weight patients with FM, overweight and obese patients with FM report greater pain severity, reduced physical function, higher levels of depression and anxiety, worse sleep quality, and poorer quality of life $[8,10,12,13]$. In addition, there are evidence to suggest that having a higher body mass index (BMI) negatively impacts exercise and physical activity participation, which in turn, often worsens the impact of the disorder [13]. Given that obesity has been implicated as a barrier to pain management [14], and similarly, pain is often described as a barrier to weight loss [15], it is plausible to suggest that weight status may be a key moderator in the successful management of FM symptomatology.

Despite accumulating evidence linking obesity and pain, relatively few studies have investigated the influence of obesity on treatment interventions in the chronic pain populations 
[16-18]. Sellinger, et al. [18] initially raised the hypothesis that obesity was associated with poor treatment outcomes, reporting that cognitive behavioral therapy was less effective in treating chronic low back pain in patients with comorbid obesity. However, no randomized controlled studies have examined the impact of obesity on the outcomes of psychological interventions for FM. Motivational Interviewing (MI) is a person-centered approach that aims to resolve an individual's ambivalence about, and improve adherence to, a specific behavior change [19]. MI has shown success in modifying several health risk behaviors (e.g. weight loss, physical inactivity) $[20,21]$ and may be a useful approach for improving treatment adherence in chronic pain conditions [22]. However, the moderating role of obesity in MI treatment effectiveness has not been tested.

The Research to Encourage Exercise for Fibromyalgia (REEF) study was a randomized clinical trial to evaluate the efficacy of MI to encourage a more physically active lifestyle in patients with FM to improve FM-relevant clinical outcomes [23]. In this study, the beneficial effects of MI on physical activity and clinical outcomes were evident immediately postintervention, but not at the 6-month follow-up. For this secondary analysis, data from the REEF study were used to address the question of whether obesity moderates MI-based treatment outcomes in patients with FM. We hypothesized that there was a significant interaction between obesity and treatment groups; specifically, that the long term effect of MI on global FM symptom severity (as measured by Fibromyalgia Impact Questionnaire/FIQ) will be attenuated in the presence of coexisting obesity.

\section{MATERIALS AND METHODS}

Experimental Design. Details of the REEF study design, inclusion and exclusion criteria, and measurement protocols have been described elsewhere [24]. Briefly, following randomization to 
either the MI intervention group or attention-control (AC) group (and prior to the MI counseling phase of the study), each patient received an individualized exercise prescription and two supervised exercise sessions. After completing both exercise sessions, participants received either six exercise-based (MI) or six FM-related health education (AC) telephone calls over the following 12 weeks. To evaluate the long-term effects of MI, phone calls (both groups) were discontinued at week 12 and follow-up assessments of our outcome measures were conducted at week 12 (immediate post-intervention), week 24 (3-month follow-up) and week 36 (6-month follow-up). The study was approved by the Indiana University Institutional Review Board in accordance with The Code of Ethics of the World Medical Association (Declaration of Helsinki). Study Participants. Patients invited to participate were referred from specialty (rheumatology, neurology, pain management) or primary care clinics with an initial diagnosis of FM. Eligibility requirements included the following: (a) male or female between 18-65 years old; (b) 1990 American College of Rheumatology classification criteria for FM [25]; and (c) Brief Pain Inventory (BPI) pain intensity score $>4$ [26]. The exclusion criteria were known cardiovascular or pulmonary disease, any musculoskeletal or neurological disorders that would preclude moderate-intensity exercise, other inflammatory rheumatic conditions (e.g. rheumatoid arthritis, systemic lupus, or other connective tissue disease), participation in moderate-vigorous physical activity on three or more days a week during the previous six months, and treatment with drugs affecting the chronotropic response to exercise (e.g. beta-blockers). Complete details of participant recruitment, including inclusion/exclusion criteria have been described previously [24].

Exercise training program. Both $\mathrm{MI}$ and AC participants received two supervised exercise sessions and an individualized exercise prescription that included the initial exercise frequency 
(2-3 d/wk), intensity (40-50\% of heart rate reserve/HRR), and duration (10-12 min/session), as well as the progression of the exercise program over the succeeding 36 weeks. All participants were instructed to gradually increase their total volume of exercise to a maximum of 55\%-65\% of HRR, 28-30 $\mathrm{min} / \mathrm{session}$, and 3-4 d/wk over the ensuing 36 weeks [23].

Primary outcome measure. The Fibromyalgia Impact Questionnaire (FIQ) is a reliable, validated self-administered questionnaire designed to assess overall symptom burden in patients with FM [27]. The FIQ contains 10 subscales (score range 0-10), which are summed to yield the total FIQ score (FIQ). Higher score on the FIQ indicate a greater overall symptom burden on the individual.

Secondary outcome measures. Pain intensity was assessed with the Brief Pain Inventory (BPI), a self-report questionnaire designed to assess the severity and overall impact of pain. The BPI rates pain severity on 4 items (current, worst, least, and average pain in past week) [26]. The BPI has been proven reliable, valid and responsive to change in patients with chronic nonmalignant pain [28].

Depression severity was assessed with the Patient Health Questionnaire 8-item Depression Scale (PHQ-8), a brief, self-administered questionnaire designed to assess major depressive disorder symptoms. The PHQ-8 allows a score (range: 0 to 24) based on the total number and severity of depressive symptoms reported over the previous two weeks [29].

Physical activity was assessed with the Community Healthy Activities Model Program for Seniors (CHAMPS), a self-report questionnaire that asks about the frequency, intensity, and duration of physical activity usually performed during a typical week over the previous four weeks. In older adults, CHAMPS has demonstrated content and construct validity, internal consistency, and responsiveness to change [30]. CHAMPS was used in the current study due to 
its ability to capture a wide range of physical activities, including household, occupational, daily tasks, and exercise. The time spent in light-moderate physical activity (PA) was calculated in accordance with the original scoring instructions for the CHAMPS questionnaire [31].

The 6-Minute Walk Test (6MWT) is a clinically relevant measure of aerobic exercise capacity that is reproducible, sensitive to change, and significantly related to the FIQ score in patients with FM $[32,33]$. For this study, the 6MWT was performed indoors on a 30-meter flat surface consistent with guidelines recommended by the American Thoracic Society [34]. Each participant completed the 6MWT at study entry (baseline), immediate post-intervention (week 12), and at the 6-month follow-up (week 36).

Statistical Analyses. BMI values were used to categorize participants as either obese (BMI > 30 $\mathrm{kg} / \mathrm{m}^{2}$ ) or non-obese $\left(\mathrm{BMI}<30 \mathrm{~kg} / \mathrm{m}^{2}\right)$. Mixed linear models were used to analyze the data, in order to determine if there were significant interactions between treatment groups and obesity. Mixed linear models allow for the addition of covariates, as well as the use of the appropriate covariance structure. The general model utilized a $2 \times 2$ factorial model, with two treatment groups (MI and AC) and two BMI categories ( $<30$ and $30+)$, and the inclusion of an interaction term and adjustment for baseline values of the variables of interest. This method also allows for the analysis of within group differences (i.e., comparing treatment groups within BMI categories) while keeping the error rate from becoming inflated. All analytic assumptions were verified and all analyses were performed using SAS v9.4 (SAS Institute, Cary, NC).

\section{RESULTS}

Of the 996 patients screened for participation in the study, 216 (22\%) met the inclusion criteria and were randomized to MI $(n=107)$ and AC $(n=109)$. Of these, 198 had complete data at last follow-up visit (retention rate $=91.6 \%$ ) and were included for analyses. Baseline 
characteristics were similar across treatment groups on all variables ( $p>0.20)$, except BMI. Compared to the AC group, participants in the MI group were slightly heavier (32.49 \pm 7.60 vs. $30.73 \pm 6.65 ; \mathrm{p}=0.08)$. Descriptive data for all participants are presented in Table 1 .

Baseline characteristics also were similar across BMI categories, except for depression (PHQ-8) and aerobic endurance capacity (6MWT). Compared to the non-obese group ( $\mathrm{n}=91$; $46 \%)$, those in the obese subset $(\mathrm{n}=107 ; 54 \%)$ had higher PHQ- 8 scores $(13.17 \pm 5.12$ vs. 11.49 $\pm 4.57 ; \mathrm{p}=0.02)$ and walked a shorter distance during the $6 \mathrm{MWT}(465.8 \pm 68.84$ meters vs. 508.1 \pm 82.32 meters; $\mathrm{p}<0.01)$.

Table 2 displays results of the interactions between treatment group assignment and BMI status across all outcomes measures. On the primary outcome measure of global FM symptom severity (i.e., FIQ), the interaction between treatment group assignment and obesity was significant $(\mathrm{p}=0.02)$. Specifically, in the non-obese group, MI was associated with a greater reduction in FIQ than AC (Figure 1A). In contrast, in the obese group, MI participants reported less reduction in FIQ compared to AC participants. On subsequent subgroup analyses, MI was superior to AC [-15.79 (2.96) vs. -8.79 (2.51), $\mathrm{p}=0.07$ ) among the non-obese, but not among the obese participants [-13.56 (2.38) vs, $-18.14(2.63), \mathrm{p}=0.19]$.

Among the secondary outcome measures, only BPI pain intensity showed statistically significant interaction between treatment group assignment and obesity $(\mathrm{p}=0.01)$. Among nonobese participants, MI was associated with greater reduction in BPI pain intensity compared to the AC group (Figure 1B). The reverse was true in the obese group. In the latter group, MI participants reported less reduction in BPI pain intensity compared to AC participants. 
Although the interaction analyses for both the 6MWT and physical activity (CHAMPS) were not statistically significant ( $\mathrm{p}=0.17$ and 0.54 , respectively), the subset analyses showed interesting findings. Among the non-obese participants, MI subjects significantly increased walking distance at the 6-month follow-up (56 \pm 10 meters) compared to the AC group ( $25 \pm 9$ meters; $\mathrm{p}=0.02$ ). In the obese group, the change in walking distance was not different between MI and $\mathrm{AC}(\mathrm{p}=0.58)$. For CHAMPS, the treatment group difference (in favor of MI over AC) with regards to the amount of light-moderate physical activity was greater among non-obese participants ( $\Delta=1.96 \pm 1.64$ hours/week) compared to the obese subset $(\Delta=0.61 \pm 1.51$ hours/week), albeit the difference was not statistically significant.

\section{DISCUSSION}

This is the first study to demonstrate that obese and non-obese participants with FM show post-treatment differences to MI, suggesting that non-obese participants respond better to MI compared to their obese counterparts. Among those receiving MI, improvement in global FM symptom severity (FIQ) and pain intensity (BPI) was greater in participants with BMI <30 $\mathrm{kg} / \mathrm{m}^{2}$. Although the interaction of treatment group assignment and obesity status was not statistically significant for physical activity (CHAMPS), the treatment group difference favoring MI (over AC) was larger among non-obese participants compared to obese participants (mean increase 2 hours/week vs. mean increase 37 minutes/week). In addition, MI was associated with a statistically significant increased walking distance, but only in the non-obese subset of the cohort.

Evidence of an association between obesity and FM continues to grow, yet the mechanisms linking obesity to chronic pain remain unclear [11]. The following obesity-related factors may contribute to increase pain or chronification of pain: musculoskeletal overload [35], 
altered body mechanics/gait [36], and systemic inflammation [37]. In addition to the these biological variables, cognitive factors may also, indirectly or directly, play a role. Several studies have shown that obesity is associated with several cognitive alterations, including negative perceptions about the benefits of exercise and physical activity [38], less enjoyment during exercise [39], more profound social stigmatization [40], and fear of movement [41]. Obese patients with FM often identify a greater number of barriers to performing health promoting behaviors and report lower self-efficacy for performing physical activity [9]. Moreover, obese persons frequently describe exercise as uncomfortable and unpleasant, which in turn, often leads to activity avoidance $[42,43]$. Thus, while exercise and physical activity have the potential to improve pain symptoms and physical function, the confluence of weight-related discomfort and additional cognitive distractors may have rendered exercise-based MI ineffective among obese participants compared to the their non-obese counterparts.

Light-moderate intensity physical activity is effective in reducing pain symptoms in patients with FM [44]. In the current study, non-obese participants receiving MI reported a greater increase in light-moderate physical activity (mean increase $\approx 2 \mathrm{hr} / \mathrm{wk}$ ) compared to obese participants receiving MI (mean increase $\approx 37 \mathrm{~min} / \mathrm{wk}$ ). Although this post-treatment difference was not statistically significant (partly due to the wide confidence intervals of CHAMPS), our findings are likely clinically relevant. Despite public health recommendation to engage in at least 150 minutes of moderate-intensity physical activity each week [45], it is becoming increasingly recognized that even small doses of physical activity (i.e. reduced frequency, intensity and/or duration) can confer significant health benefits [46]. A recent systemic review of older adults has found that low intensity exercise is effective in improving several aspects of health pertinent to patients with FM, including muscular strength, flexibility, balance, depressive 
symptoms, and fall risk [47]. Furthermore, previous research has shown that increase exercise capacity is predictive of improvement in cognition [48], and that exercise bouts as short as 10 minutes are associated with significant improvement in psychological distress [49]. In light of this evidence, we speculate that MI may have been more effective in improving psychological outlook and motivation to be more physically active in non-obese patients with FM. This inference is strengthened by our finding that MI was associated with a significantly greater increase in exercise capacity (6MWT) in non-obese participants $(\mathrm{p}=0.02)$ compared to their obese counterparts $(\mathrm{p}=0.58)$.

The results of this study must be considered in the context of several limitations. First, given that the current report was a post-hoc analysis of a previously completed randomized clinical trial, it is possible that our study findings could have occurred by chance alone and/or from a sample size that was inadequate to fully assess interaction effects. However, there are biological and psychological models to support the moderating effect of obesity on treatment success. Second, our study population consisted primarily of women reporting higher levels of pain. Thus, the extent to which these findings extend to men with FM, or those reporting less severe pain symptoms, is unclear. However, the clinical characteristics of the current study participants are comparable to other psychoeducational-based clinical trials in FM [50]. Finally, the present study focused solely on the influence of obesity and not on other comorbid conditions commonly associated with excessive weight gain (e.g. cardiovascular disease, chronic lung disease, depression). Given that the current study excluded subjects with cardiovascular disease and chronic lung disease, the moderating effects of these two medical conditions cannot be evaluated. Interestingly, depression, another common comorbid condition in FM, was not a treatment moderator in our study (data not shown). Despite these limitations, the strengths of this 
study include its longitudinal design, large sample size, longer study duration, and low dropout rate $(18 / 216=8.3 \%)$.

\section{CONCLUSION}

This is the first study to investigate the influence of obesity on a psychological intervention designed to improve clinical outcomes in FM. Our findings suggest that obesity negatively influences the ability of MI to improve global FM symptom severity and pain, and may contribute to reduced physical activity, in patients with FM. Obesity that coexists with FM may serve as a barrier to the effectiveness of MI designed to improve physical activity and clinical outcomes in individuals with FM. Future studies should examine the clinical effectiveness (including cost benefit analyses) of behavioral interventions that combine physical activity and weight management counseling on clinical outcomes in patients with FM. Although our study findings need to be replicated in larger, more diverse samples, the results from this study can assist clinician decision-making regarding the role of exercise-based MI in the treatment algorithm for patients with FM.

Acknowledgement

The authors wish to thank Engy Missaiel, MD for help in writing the manuscript.

Copyright (C) 2017 Wolters Kluwer Health, Inc. Unauthorized reproduction of the article is prohibited. 


\section{REFERENCES}

1. Lachaine J, Beauchemin C and Landry PA. Clinical and economic characteristics of patients with fibromyalgia syndrome. Clin J Pain 2010;26:284-90.

2. Panton LB, Kingsley JD, Toole T, et al. A comparison of physical functional performance and strength in women with fibromyalgia, age- and weight-matched controls, and older women who are healthy. Phys Ther 2006;86:1479-88.

3. Weir PT, Harlan GA, Nkoy FL, et al. The incidence of fibromyalgia and its associated comorbidities: a population-based retrospective cohort study based on International Classification of Diseases, 9th Revision codes. J Clin Rheumatol 2006;12:124-8.

4. Przekop P, Haviland MG, Zhao Y, et al. Self-reported physical health, mental health, and comorbid diseases among women with irritable bowel syndrome, fibromyalgia, or both compared with healthy control respondents. J Am Osteopath Assoc 2012;112:726-35.

5. McLoughlin MJ, Colbert LH, Stegner AJ, et al. Are women with fibromyalgia less physically active than healthy women? Med Sci Sports Exerc 2011;43:905-12.

6. Neumann L, Lerner E, Glazer Y, et al. A cross-sectional study of the relationship between body mass index and clinical characteristics, tenderness measures, quality of life, and physical functioning in fibromyalgia patients. Clin Rheumatol 2008;27:1543-7.

7. Okifuji A, Donaldson GW, Barck L, et al. Relationship between fibromyalgia and obesity in pain, function, mood, and sleep. J Pain 2010;11:1329-37.

8. Aparicio VA, Ortega FB, Carbonell-Baeza A, et al. Relationship of weight status with mental and physical health in female fibromyalgia patients. Obes Facts 2011;4:443-8.

9. Timmerman GM, Calfa NA and Stuifbergen AK. Correlates of body mass index in women with fibromyalgia. Orthop Nurs 2013;32:113-9.

Copyright (C) 2017 Wolters Kluwer Health, Inc. Unauthorized reproduction of the article is prohibited. 
10. Gota CE, Kaouk S and Wilke WS. Fibromyalgia and Obesity: The Association Between Body Mass Index and Disability, Depression, History of Abuse, Medications, and Comorbidities. J Clin Rheumatol 2015;21:289-95.

11. Okifuji A and Hare BD. The association between chronic pain and obesity. J Pain Res 2015;8:399-408.

12. de Araujo TA, Mota MC and Crispim CA. Obesity and sleepiness in women with fibromyalgia. Rheumatol Int 2015;35:281-7.

13. Vincent A, Clauw D, Oh TH, et al. Decreased physical activity attributable to higher body mass index influences fibromyalgia symptoms. PM R 2014;6:802-7.

14. Mauro M, Taylor V, Wharton S, et al. Barriers to obesity treatment. Eur J Intern Med 2008;19:173-80.

15. Wachholtz A, Binks M, Eisenson H, et al. Does pain predict interference with daily functioning and weight loss in an obese residential treatment-seeking population? Int J Behav Med 2010;17:118-24.

16. Wertli MM, Held U, Campello M, et al. Obesity is associated with more disability at presentation and after treatment in low back pain but not in neck pain: findings from the OIOC registry. BMC Musculoskelet Disord 2016;17:140.

17. Cuesta-Vargas AI and Gonzalez-Sanchez M. Obesity effect on a multimodal physiotherapy program for low back pain suffers: patient reported outcome. J Occup Med Toxicol 2013;8:13. 18. Sellinger JJ, Clark EA, Shulman M, et al. The moderating effect of obesity on cognitivebehavioral pain treatment outcomes. Pain Med 2010;11:1381-90.

19. Miller WR and Rollnick S. Motivational interviewing : preparing people to change addictive behavior. New York: Guilford Press, 1991.

Copyright (C) 2017 Wolters Kluwer Health, Inc. Unauthorized reproduction of the article is prohibited. 
20. O'Halloran PD, Blackstock F, Shields N, et al. Motivational interviewing to increase physical activity in people with chronic health conditions: a systematic review and meta-analysis. Clin Rehabil 2014;28:1159-71.

21. Tse MM, Vong SK and Tang SK. Motivational interviewing and exercise programme for community-dwelling older persons with chronic pain: a randomised controlled study. J Clin Nurs 2013;22:1843-56.

22. Alperstein D and Sharpe L. The Efficacy of Motivational Interviewing in Adults With Chronic Pain: A Meta-Analysis and Systematic Review. J Pain 2016;17:393-403.

23. Ang DC, Kaleth AS, Bigatti S, et al. Research to encourage exercise for fibromyalgia (REEF): use of motivational interviewing, outcomes from a randomized-controlled trial. Clin J Pain 2013;29:296-304.

24. Ang DC, Kaleth AS, Bigatti S, et al. Research to Encourage Exercise for Fibromyalgia (REEF): use of motivational interviewing design and method. Contemp Clin Trials 2011;32:5968.

25. Wolfe F, Smythe HA, Yunus MB, et al. The American College of Rheumatology 1990 Criteria for the Classification of Fibromyalgia. Report of the Multicenter Criteria Committee. Arthritis Rheum 1990;33:160-72.

26. Cleeland CS and Ryan KM. Pain assessment: global use of the Brief Pain Inventory. Ann Acad Med Singapore 1994;23:129-38.

27. Mannerkorpi K, Ahlmen M and Ekdahl C. Six- and 24-month follow-up of pool exercise therapy and education for patients with fibromyalgia. Scand J Rheumatol 2002;31:306-10. 28. Tan G, Jensen MP, Thornby JI, et al. Validation of the Brief Pain Inventory for chronic nonmalignant pain. J Pain 2004;5:133-7.

Copyright (C) 2017 Wolters Kluwer Health, Inc. Unauthorized reproduction of the article is prohibited. 
29. Thombs BD, Benedetti A, Kloda LA, et al. The diagnostic accuracy of the Patient Health Questionnaire-2 (PHQ-2), Patient Health Questionnaire-8 (PHQ-8), and Patient Health Questionnaire-9 (PHQ-9) for detecting major depression: protocol for a systematic review and individual patient data meta-analyses. Syst Rev 2014;3:124.

30. Harada ND, Chiu V, King AC, et al. An evaluation of three self-report physical activity instruments for older adults. Med Sci Sports Exerc 2001;33:962-70.

31. Stewart AL, Mills KM, King AC, et al. CHAMPS physical activity questionnaire for older adults: outcomes for interventions. Med Sci Sports Exerc 2001;33:1126-41.

32. Pankoff B, Overend T, Lucy D, et al. Validity and responsiveness of the 6 minute walk test for people with fibromyalgia. J Rheumatol 2000;27:2666-70.

33. King S, Wessel J, Bhambhani Y, et al. Validity and reliability of the 6 minute walk in persons with fibromyalgia. J Rheumatol 1999;26:2233-7.

34. American Thoracic Society. ATS Statement: Guidelines for the Six-Minute Walk Test. Am J Respir Crit Care Med. 2002:111-7.

35. Singh D, Park W, Hwang D, et al. Severe obesity effect on low back biomechanical stress of manual load lifting. Work 2015;51:337-48.

36. Cimolin V, Vismara L, Galli M, et al. Effects of obesity and chronic low back pain on gait. $J$ Neuroeng Rehabil 2011;8:55.

37. Ronti T, Lupattelli G and Mannarino E. The endocrine function of adipose tissue: an update. Clin Endocrinol (Oxf) 2006;64:355-65.

38. Tsuritani I, Honda R, Noborisaka Y, et al. Impact of obesity on musculoskeletal pain and difficulty of daily movements in Japanese middle-aged women. Maturitas 2002;42:23-30.

Copyright (C) 2017 Wolters Kluwer Health, Inc. Unauthorized reproduction of the article is prohibited. 
39. Leone LA and Ward DS. A mixed methods comparison of perceived benefits and barriers to exercise between obese and nonobese women. J Phys Act Health 2013;10:461-9.

40. Ekkekakis P, Lind E and Vazou S. Affective responses to increasing levels of exercise intensity in normal-weight, overweight, and obese middle-aged women. Obesity (Silver Spring) 2010;18:79-85.

41. Vincent HK, Omli MR, Day T, et al. Fear of movement, quality of life, and self-reported disability in obese patients with chronic lumbar pain. Pain Med 2011;12:154-64.

42. Ekkekakis P, Vazou S, Bixby WR, et al. The mysterious case of the public health guideline that is (almost) entirely ignored: call for a research agenda on the causes of the extreme avoidance of physical activity in obesity. Obes Rev 2016;17:313-29.

43. Kruger J, Yore MM and Kohl HW, 3rd. Leisure-time physical activity patterns by weight control status: 1999-2002 NHANES. Med Sci Sports Exerc 2007;39:788-95.

44. Hauser W, Klose P, Langhorst J, et al. Efficacy of different types of aerobic exercise in fibromyalgia syndrome: a systematic review and meta-analysis of randomised controlled trials. Arthritis Res Ther 2010;12:R79.

45. Garber CE, Blissmer B, Deschenes MR, et al. American College of Sports Medicine Position Stand. Quantity and quality of exercise for developing and maintaining cardiorespiratory, musculoskeletal, and neuromotor fitness in apparently healthy adults: guidance for prescribing exercise. Med Sci Sports Exerc 2011;43:1334-59.

46. Wasfy MM and Baggish AL. Exercise Dose in Clinical Practice. Circulation 2016;133:2297313.

47. Tse AC, Wong TW and Lee PH. Effect of Low-intensity Exercise on Physical and Cognitive Health in Older Adults: a Systematic Review. Sports Med Open 2015;1:37.

Copyright (C) 2017 Wolters Kluwer Health, Inc. Unauthorized reproduction of the article is prohibited. 
48. Vidoni ED, Johnson DK, Morris JK, et al. Dose-Response of Aerobic Exercise on Cognition: A Community-Based, Pilot Randomized Controlled Trial. PLoS One 2015;10:e0131647.

49. Sullivan AB, Covington E and Scheman J. Immediate benefits of a brief 10-minute exercise protocol in a chronic pain population: a pilot study. Pain Med 2010;11:524-9.

50. Glombiewski JA, Sawyer AT, Gutermann J, et al. Psychological treatments for fibromyalgia: a meta-analysis. Pain 2010;151:280-95.

Copyright (C) 2017 Wolters Kluwer Health, Inc. Unauthorized reproduction of the article is prohibited. 


\section{Demographics}

Age in years

Gender, \% female

Ethnicity, \% non-Hispanic

Race, \% white

Education, $\%>$ high school

Marital status, $\%$ married

Employment, \% employed

\section{Clinical Variables}

Body mass index $>=30\left(\mathrm{~kg} / \mathrm{m}^{2}\right) \%$

Duration of fibromyalgia diagnosis (years)

PHQ-8 depression (range 0-24)†

BPI pain severity (range $0-10) \dagger$

FIQ (range 0-100)†

Medications, $\%$ prescribed

Non-tricyclic antidepressants

Anticonvulsants

Opioid analgesics

\section{Physical Activity Measures}

\section{CHAMPS}

Light-to-moderate physical activity

(hours/week)

6-minute walk test (meters) 60.8

$46.2(11.0)$

$45.8(11.2)$

95.9

95.1

99.0

98.0

90.7

85.2

77.3

58.8

58.8

81.2

63.4

47.5

47.5

$9.3(7.5)$

$12.6(5.1)$

$6.0(1.4)$

$66.9(11.7)$

$66.7(13.4)$

56.4

27.7

34.7
$9.1(6.4)$

$12.2(4.8)$

$5.9(1.2)$

66.0

29.9

34.0

*Values are the means (standard deviation) unless otherwise indicated.

$\dagger$ Higher score indicates a worse state of health.

Abbreviations: PHQ-8 = Patient Health Questionnaire-8; BPI = Brief Pain Inventory; FIQ = Fibromyalgia Impact Questionnaire; CHAMPS = Community Health Activities Model Program for Seniors; LMPA= light-to-moderate physical activity. $\mathrm{n}=$ number of patients 
Table 2. Interactions between Treatment Group Assignment and Body Mass Index

\begin{tabular}{|c|c|c|c|c|c|}
\hline & $\begin{array}{l}\text { Motivational } \\
\text { Interviewing } \\
\text { (MI) }\end{array}$ & $\begin{array}{l}\text { Attention } \\
\text { Control (AC) }\end{array}$ & $\begin{array}{l}\text { p-value } \\
\text { (MI vs. AC) }\end{array}$ & $\begin{array}{l}\text { Treatment Difference } \\
\text { Mean (95\% confidence } \\
\text { interval) }\end{array}$ & $\begin{array}{l}\text { p-value } \\
\text { (Interaction } \\
\text { between } \\
\text { treatment and } \\
\text { obesity) } \\
\end{array}$ \\
\hline \multicolumn{6}{|l|}{ Body mass index $<30$} \\
\hline \multicolumn{6}{|l|}{ Primary outcome } \\
\hline FIQ & $-15.79(2.96)$ & $-8.79(2.51)$ & 0.07 & $\begin{array}{l}7.01 \\
(-0.64,14.66)\end{array}$ & $0.02 *$ \\
\hline \multicolumn{6}{|l|}{ Secondary outcomes } \\
\hline BPI pain & $-1.52(0.29)$ & $-0.87(0.25)$ & 0.08 & $\begin{array}{l}0.65 \\
(-0.09,1.40)\end{array}$ & $0.01 *$ \\
\hline 6-MWT (meters) & $56.14(9.88)$ & $25.19(8.92)$ & $0.02 *$ & $\begin{array}{l}30.94 \\
(4.92,5\end{array}$ & 0.17 \\
\hline $\begin{array}{l}\text { Light-to- moderate } \\
\text { Physical activity } \\
\text { (hours/week) }\end{array}$ & $4.19(1.25)$ & $2.22(1.06)$ & 0.23 & & 0.54 \\
\hline \multicolumn{6}{|c|}{ Body mass index $>=30$} \\
\hline \multicolumn{6}{|l|}{ Primary outcome } \\
\hline FIQ & $-13.56(2.38)$ & $-18.14(2.63)$ & & $\begin{array}{l}4.58 \\
(-2.41,11.57)\end{array}$ & \\
\hline \multicolumn{6}{|l|}{ Secondary outcomes } \\
\hline BPI pain & $-1.10(0.23)$ & $-1.69(0.26)$ & & $\begin{array}{l}0.59 \\
(-0.09,1.27)\end{array}$ & \\
\hline 6-MWT (meters) & $33.38(8.40)$ & $26.70(8.98$ & & $\begin{array}{l}6.68 \\
(-17.44,30.80)\end{array}$ & \\
\hline $\begin{array}{l}\text { Light-to-moderate } \\
\text { Physical activity } \\
\text { (hours/week) }\end{array}$ & $3.74(1.01)$ & $3.13(1.12)$ & 0. & $\begin{array}{l}0.61 \\
(-2.36,3.59)\end{array}$ & \\
\hline
\end{tabular}

Values are mean (standard error) change from baseline, adjusting for baseline value.

Treatment difference: mean treatment difference (95\% confidence interval)

FIQ = Fibromyalgia Impact Questionnaire; BPI = Brief Pain Inventory; 6-MWT= 6 minute walk test $* \mathrm{p}<0.05$

Copyright (C) 2017 Wolters Kluwer Health, Inc. Unauthorized reproduction of the article is prohibited. 


\section{Figure Legends.}

Figure 1. Treatment Outcomes based on Obesity Status

Values represent mean change with standard error bar. For FIQ and BPI= the more negative the change in values, the greater the improvement. For 6MWT and Low-Mod PA= the more positive the change in values, the greater the improvement.

FIQ: Fibromyalgia Impact Questionnaire; BPI: Brief Pain Inventory; 6MWT: 6 minute walk test; Low-Mod PA: self reported light to moderate physical activity (CHAMPS)

Copyright (C) 2017 Wolters Kluwer Health, Inc. Unauthorized reproduction of the article is prohibited. 

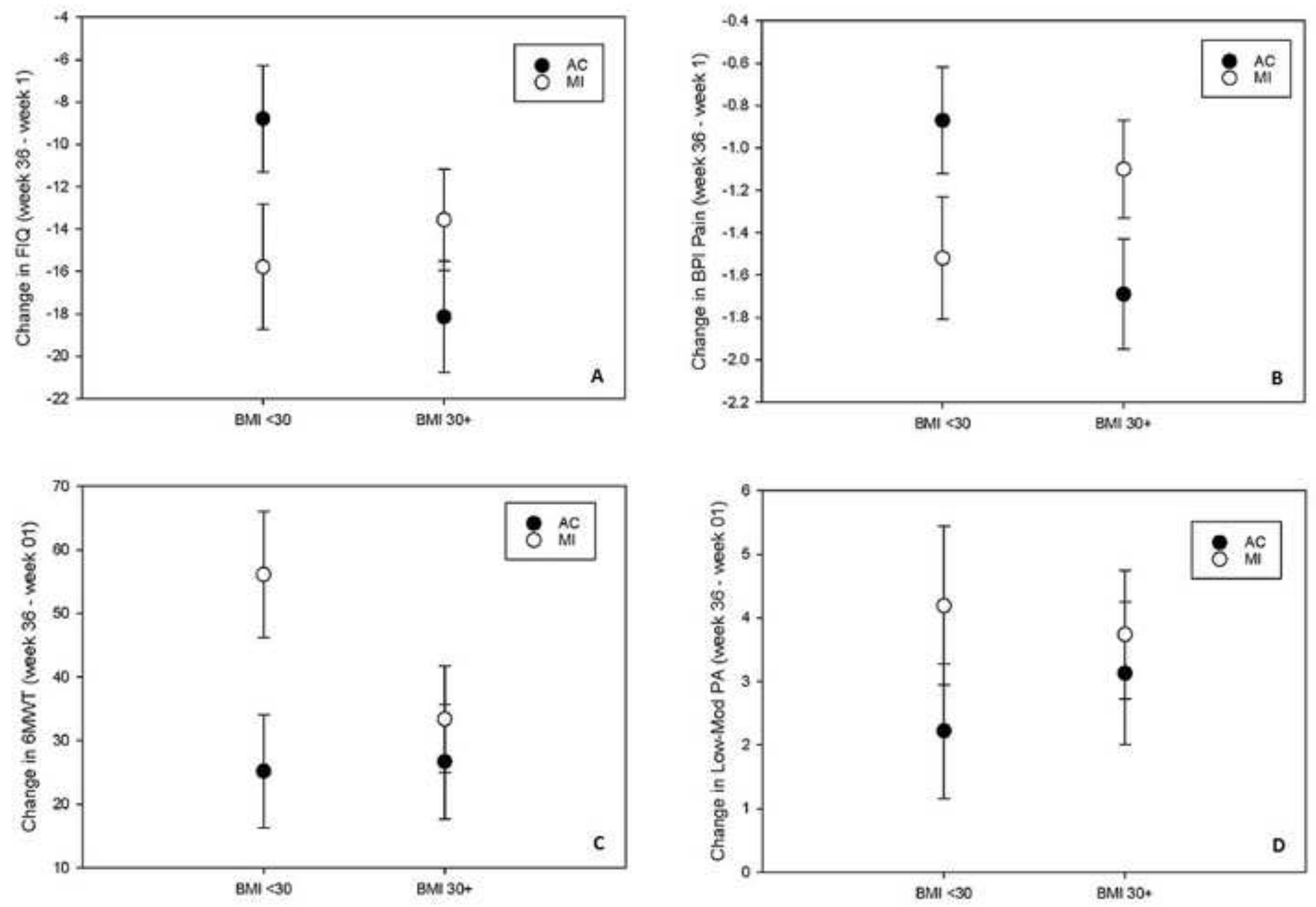

Copyright (C) 2017 Wolters Kluwer Health, Inc. Unauthorized reproduction of the article is prohibited. 